\title{
Structures on the Product of Two Almost Hermitian Almost Contact Manifolds
}

\author{
Gherici Beldjilali* and Mohamed Belkhelfa \\ (Communicated by Ion Mihai)
}

\begin{abstract}
The purpose of this paper is to define some classes of almost contact metric 3-structures manifolds and almost quaternionic metric with an almost Hermitian almost contact metric structure. Next, we construct an almost quaternionic Hermitian structure on the product of two almost Hermitian almost contact metric structures. This gives a new positive answer to a question raised by $T$. Tshikuna-Matamba [7].
\end{abstract}

Keywords: Almost Hermitian almost contact structure, almost contact metric manifolds with 3-structures, hyperkählerian structure.

AMS Subject Classification (2010): Primary: 53D15 ; Secondary: 53C26

\section{Introduction}

By means of a natural change of the product metric, one can construct widely almost Hemitian structures on product manifolds of two almost contact metric manifolds. Since many almost contact metric and Sasakian structures are now found (for example, see [1], [?] ), this method enables us to provide various kinds of almost Hermitian structures.

On the other hand, by deforming the canonical almost Hermitian structure with some functions of the norm of vectors, one can construct many geometric structures of new type (almost Hermitian and Kähler structures, almost quaternionic Hermitian, etc.) on the tangent bundles of Riemannian and almost Hermitian manifolds (see [7], [9]).

Watanabe ([13],[14]) survey almost Hermitian, Kähler, almost quaternionic Hermitian and quaternionic Kähler structures, naturally constructed on products of manifolds with almost contact metric and Sasakian structures and open intervals. As an application of these constructions. Next, he investigate almost Hermitian structures, naturally defined on the product manifolds of two almost contact metric and Sasakian manifolds, and he study almost quaternionic Hermitian and quaternionic Kähler structures on products of 3-Sasakian manifolds and open intervals then he ask some problems related to these topics.

In [11], Tshikuna-Matamba determined some remarkable classes of the induced structures on the product of two Riemannian manifolds. He deal with the product of an almost quaternion manifold with an almost Hermitian almost contact metric manifold. This product has been used to construct other classes of almost contact metric manifolds with 3-structures in [10], and he end the study with some problems.

This paper is organized in the following way.

Section 2 is devoted to the background of the manifolds which will be used in the sequel.

Section 3 is devoted to construct a family of almost contact metric 3-structures manifolds from an almost Hermitian almost contact metric structure.

Section 4 is devoted to construct a family of almost quaternionic metric manifolds from an almost Hermitian almost contact metric structure.

Section 5 we deal the product of two almost Hermitian almost contact metric structures. 


\section{Preliminaries on manifolds}

Recall that an almost Hermitian manifold is a Riemannian manifold $(M, g)$ equipped with a tensor field $J$ of type $(1,1)$ such that for all vectors fiels $X, Y$ on $M$ the following two conditions are satisfied:

$$
J^{2}(X)=-X, \quad g(J X, J Y)=g(X, Y) .
$$

An almost complex structure $J$ is integrable, and hence the manifold is a complex manifold, if and only if its Nijenhuis tensor $N_{j}$ vanishes, with

$$
N_{j}(X, Y)=[J X, J Y]-[X, Y]-J[X, J Y]-J[J X, Y] .
$$

Any almost Hermitian manifold $(M, g, J)$ possesses a differential 2-form $\Omega$, called the fundamental 2-form or the Kähler 2-form, defined by

$$
\Omega(X, Y)=g(X, J Y) .
$$

$(M, J, g)$ is then called almost Kähler if $\Omega$ is closed i.e. $d \Omega=0$. An almost Kähler manifold with integrable $J$ is called a Kähler manifold, and thus is characterized by the conditions: $d \Omega=0$ and $N=0$. One can prove that these both conditions combined are equivalent with the single condition

$$
\nabla J=0 .
$$

An almost quaternionic metric manifold is a quintuple $\left(M, g, J_{1}, J_{2}, J_{3}\right)$, where

$$
\left\{\begin{array}{l}
(1):(M, g) \text { is a Riemannian manifold; } \\
(2):\left(g, J_{i}\right) \text { is an almost Hermitian structure on } M \text { for } i=1,2,3 \\
(3): J_{1} J_{2}=J_{3}, J_{2} J_{3}=J_{1}, J_{3} J_{1}=J_{2} .
\end{array}\right.
$$

Almost quaternionic metric manifolds are of dimension $4 m$ and their nomenclature is related to that of almost Hermitian structures. According Calabi [2], for a structure to be hyperkählerian, it is sufficient that in $\left(g, J_{1}, J_{2}, J_{3}\right)$ two of these structures are Kählerians. A differential 4 -form is defined by

$$
\tilde{\Omega}=\sum_{i=1}^{3} \Omega_{i} \wedge \Omega_{i}
$$

An almost quaternion metric manifold is quaternion Kählerian manifold if and only if $\nabla \tilde{\Omega}=0$ [15].

By an almost contact metric manifold, one understands a quintuple $(M, g, \varphi, \xi, \eta)$, where

(1) $\xi$ is a characteristic vector field ;

(2) $\eta$ is a differential 1-form such that $\eta(\xi)=1$;

(3) $\varphi$ is a tensor field of type $(1,1)$ satisfying $\varphi^{2} X=-X+\eta(X) \xi$;

(4) $g(\varphi X, \varphi Y)=g(X, Y)-\eta(X) \eta(Y)$.

Replacing $J$ by $\varphi$, the fundamental 2 -form $\phi$ is defined by

$$
\phi(X, Y)=g(X, \varphi Y) .
$$

Denoting by $\nabla$ the Levi-Civita connection of $g$, the covariant derivative of $\eta$ and the exterior differential of $\eta$ are defined, respectively, by

$$
\begin{gathered}
\left(\nabla_{X} \eta\right) Y=g\left(Y, \nabla_{X} \xi\right), \\
2 d \eta(X, Y)=\left(\nabla_{X} \eta\right) Y-\left(\nabla_{Y} \eta\right) X,
\end{gathered}
$$

An almost contact metric manifold is said to be almost cosymplectic if the forms $\phi$ and $\eta$ are closed, that is, $d \phi=d \eta=0$.

Such a manifold is said to be a contact metric manifold if $d \eta=\phi$. If, in addition, $\xi$ is a Killing vector field, then $M$ is said to be a K-contact manifold. It is well-known that a contact metric manifold is a K-contact manifold if and only if $\nabla_{X} \xi=-\varphi X$, for any vector field $X$ on $M$. On the other hand, the almost contact metric structure 
of $M$ is said to be normal if $[\varphi, \varphi](X, Y)=-2 d \eta(X, Y) \xi$, for any $X$ and $Y$ where $[\varphi, \varphi]$ denotes the Nijenhuis torsion of $\varphi$, given by

$$
[\varphi, \varphi](X, Y)=\varphi^{2}[X, Y]+[\varphi X, \varphi Y]-\varphi[\varphi X, Y]-\varphi[X, \varphi Y] .
$$

A normal almost cosymplectic manifold is called a cosymplectic manifold. It is well-known that a necessary and sufficient condition for $M$ to be cosymplectic is $\nabla \varphi=0$.

A normal contact metric manifold is called a Sasakian manifold. It can be proved that a Sasakian manifold is $\mathrm{K}$-contact, and that an almost contact metric manifold is Sasakian if and only if

$$
\left(\nabla_{X} \varphi\right) Y=g(X, Y) \xi-\eta(Y) X,
$$

for any $X, Y$. Moreover, for a Sasakian manifold have following identities:

$$
\nabla_{X} \xi=-\varphi X, \quad\left(\nabla_{X} \eta\right)(Y)=-g(\varphi X, Y) .
$$

Let $\left(\varphi_{i}, \xi_{i}, \eta_{i}\right)_{i=1}^{3}$ be three almost contact structures such that each of them is compatible with the Riemannian structure $g$ ( i.e. $\left.g\left(\varphi_{i} X, \varphi_{i} Y\right)=g(X, Y)-\eta_{i}(X) \eta_{i}(Y), i=1,2,3\right)$. We say that $\left(M, g,\left(\varphi_{i}, \xi_{i}, \eta_{i}\right)_{i=1}^{3}\right)$ is an almost contact metric manifold 3-structure if for any cyclic permutation $(i, j, k)$ of $\{1,2,3\}$ the following conditions are satisfied :

$$
\left\{\begin{array}{l}
(1): \eta_{i}\left(\xi_{j}\right)=\eta_{j}\left(\xi_{i}\right)=0 ; \\
(2): \varphi_{i} \xi_{j}=-\varphi_{j} \xi_{i}=\xi_{k} ; \\
(3): \varphi_{i} \circ \varphi_{j}-\eta_{j} \otimes \xi_{i}=-\varphi_{j} \circ \varphi_{i}+\eta_{i} \otimes \xi_{j}=\varphi_{k} ; \\
(4): \eta_{i} \circ \varphi_{j}=-\eta_{j} \circ \varphi_{i}=\eta_{k} .
\end{array}\right.
$$

Almost contact metric manifolds 3 -structure are of odd dimension $4 m+3$. When from $\left(\varphi_{i}, \xi_{i}, \eta_{i}\right)_{i=1}^{3}$ two structure at least are from class $P$, we say that $\left(\varphi_{i}, \xi_{i}, \eta_{i}\right)_{i=1}^{3}$ is in this class. In other words, a 3-Sasakian structures means that of the $\left(\varphi_{i}, \xi_{i}, \eta_{i}\right)_{i=1}^{3}$ two structures are at least Sasakian [5].

Let $(M, g, J)$ be an almost Hermitian manifold furnished with two almost contact structures $\left(\varphi_{i}, \xi_{i}, \eta_{i}\right)_{i=1}^{2}$. We say that $\left(M, g, J,\left(\varphi_{i}, \xi_{i}, \eta_{i}\right)_{i=1}^{2}\right)$ is an almost Hermitian almost contact metric manifold if for all $X, Y$ on $M$

$$
\left\{\begin{array}{l}
(1): g\left(\varphi_{i} X, Y\right)=-g\left(X, \varphi_{i} Y\right) \\
(2): J \xi_{1}=-\xi_{2}, J \xi_{2}=\xi_{1} \\
(3): \varphi_{i}^{2} X=-X+\eta_{1}(X) \xi_{1}+\eta_{2}(X) \xi_{2} \\
(4): \varphi_{1}(J X)=-J \varphi_{1} X=\varphi_{2} X \\
(5): \varphi_{2}(J X)=-J \varphi_{2} X=-\varphi_{1} X \\
(6): \varphi_{2}\left(\varphi_{1} X\right)=-\varphi_{1}\left(\varphi_{2} X\right)=J X+\eta_{1}(X) \xi_{2}-\eta_{2}(X) \xi_{1} .
\end{array}\right.
$$

The dimension of this type of manifold is $4 m+2$. For the nomenclature, we will indicate the name of the almost contact metric structure followed by the name of the almost Hermitian one. For instance, a SasakianKählerian manifold is a Kählerian manifold equipped with two Sasakian structures (see [4]).

\section{Product $M^{4 m+2} \times \mathbb{R}$}

Let $\left(M^{4 m+2}, g, J,\left(\varphi_{i}, \xi_{i}, \eta_{i}\right)_{i=1}^{2}\right)$ be an almost Hermitian almost contact metric manifold. The product $\bar{M}=$ $M^{4 m+2} \times \mathbb{R}$, has dimension $4 m+3$. We define a three almost contact structures $\left(\bar{\varphi}_{i}, \bar{\xi}_{i}, \bar{\eta}_{i}\right)_{i=1}^{3}$ by;

$$
\begin{array}{lll}
\bar{\varphi}_{1}\left(X, f \frac{\partial}{\partial r}\right)=\left(\varphi_{1} X-f \xi_{1}, \eta_{1}(X) \frac{\partial}{\partial r}\right), & \bar{\xi}_{1}=\xi_{2}, & \bar{\eta}_{1}=\eta_{2}, \\
\bar{\varphi}_{2}\left(X, f \frac{\partial}{\partial r}\right)=\left(-\varphi_{2} X+f \xi_{2},-\eta_{2}(X) \frac{\partial}{\partial r}\right), & \bar{\xi}_{2}=\xi_{1}, & \bar{\eta}_{2}=\eta_{1}, \\
\bar{\varphi}_{3}\left(X, f \frac{\partial}{\partial r}\right)=(J X, 0), & \bar{\xi}_{3}=\frac{\partial}{\partial r}, & \bar{\eta}_{3}=d r,
\end{array}
$$

and we define a Riemannian metric $\bar{g}$ by

$$
\bar{g}\left(\left(X, f \frac{\partial}{\partial r}\right),\left(Y, h \frac{\partial}{\partial r}\right)\right)=g(X, Y)+f h,
$$

for any vectors fields $X, Y$ of $M$ and $f, h$ two functions on $\mathbb{R}$. 
Proposition 3.1. The structure $\left(\bar{g},\left(\bar{\varphi}_{i}, \bar{\xi}_{i}, \bar{\eta}_{i}\right)_{i=1}^{3}\right)$ constructed as above is an almost contact metric 3-structure.

Proof. Obvious. ( using (2.8)).

The manifold $\left(\bar{M}, \bar{g},\left(\bar{\varphi}_{i}, \bar{\xi}_{i}, \bar{\eta}_{i}\right)_{i=1}^{3}\right)$ possesses a fundamental 2 -form $\bar{\phi}_{i}$; defined by:

$$
\left\{\begin{array}{l}
\bar{\phi}_{1}=\phi_{1}-\eta_{1} \wedge d r \\
\bar{\phi}_{2}=-\phi_{2}+\eta_{2} \wedge d r \\
\bar{\phi}_{3}=\Omega
\end{array}\right.
$$

Let $\nabla$ and $\bar{\nabla}$ denote the Riemannian connections of $g$ and $\bar{g}$ respectively. Now taking $X$ and $Y$ as vector fields tangent to $M$ and using algebraic properties of almost Hermitian almost contact metric and almost contact metric 3-structure given above we get,

$$
\left\{\begin{aligned}
\left(\bar{\nabla}_{\left(X, f \frac{\partial}{\partial r}\right.} \bar{\varphi}_{1}\right)\left(Y, h \frac{\partial}{\partial r}\right) & =\left(\left(\nabla_{X} \varphi_{1}\right) Y-h \nabla_{X} \xi_{1},\left(\nabla_{X} \eta_{1}\right)(Y) \frac{\partial}{\partial r}\right) \\
\left(\bar{\nabla}_{\left(X, f \frac{\partial}{\partial r}\right)} \bar{\varphi}_{2}\right)\left(Y, h \frac{\partial}{\partial r}\right) & =\left(-\left(\nabla_{X} \varphi_{2}\right) Y+h \nabla_{X} \xi_{2},-\left(\nabla_{X} \eta_{2}\right)(Y) \frac{\partial}{\partial r}\right) \\
\left(\bar{\nabla}_{\left(X, f \frac{\partial}{\partial r}\right)} \bar{\varphi}_{3}\right)\left(Y, h \frac{\partial}{\partial r}\right) & =\left(\left(\nabla_{X} J\right) Y, 0\right)
\end{aligned}\right.
$$

and we can state the following:

Proposition 3.2. Let $\left(M^{4 m+2}, g, J,\left(\varphi_{i}, \xi_{i}, \eta_{i}\right)_{i=1}^{2}\right)$ be an almost Hermitian almost contact metric manifold. If $\left(\bar{g},\left(\bar{\varphi}_{i}, \bar{\xi}_{i}, \bar{\eta}_{i}\right)_{i=1}^{3}\right)$ is an almost contact metric 3 -structure on the manifolds $\bar{M}=M \times \mathbb{R}$, constructed as above, then:

(a) $\bar{M}$ is almost 3-cosymplectic if, and only if, $M$ is almost cosymplectic-almost Kählerian;

(b) $\bar{M}$ is 3-cosymplectic if, and only if, $M$ is cosymplectic-Kählerian.

Proof. Using the definition of an almost cosymplectic structure and (3.1), The first assertion (a) is obviously. For (b), suppose that $\left(M^{4 m+2}, g, J,\left(\varphi_{i}, \xi_{i}, \eta_{i}\right)_{i=1}^{2}\right)$ is cosymplectic-Kählerian i.e. for $i=\overline{1,2}$ we have from (4.2)

$$
\left\{\begin{array}{l}
\left(\nabla_{X} \varphi_{i}\right) Y=0 \\
\left(\nabla_{X} J\right) Y=0
\end{array}\right.
$$

As it is known, $\nabla \varphi_{i}=0 \Leftrightarrow \nabla \eta_{i}=\nabla \phi_{i}=0$ and using (2.4) then $\bar{\nabla} \bar{\varphi}_{i}=0$.

Coversely, suppose that $\left(\bar{g},\left(\bar{\varphi}_{i}, \bar{\xi}_{i}, \bar{\eta}_{i}\right)_{i=1}^{3}\right)$ is 3-cosymplectic i.e. for $i=\overline{1,3}$

$$
\left(\bar{\nabla} \bar{\varphi}_{i}\right)_{\left(X, f \frac{\partial}{\partial r}\right)}\left(Y, h \frac{\partial}{\partial r}\right)=(0,0),
$$

then from (4.2) we get for $i=\overline{1,2}$

$$
\left\{\begin{array} { l } 
{ ( \nabla _ { X } \varphi _ { i } ) Y - h \nabla _ { X } \xi _ { i } = 0 } \\
{ ( \nabla _ { X } \eta _ { i } ) ( Y ) = 0 } \\
{ ( \nabla _ { X } J ) Y = 0 }
\end{array} \Rightarrow \left\{\begin{array}{l}
\left(\nabla_{X} \varphi_{i}\right) Y=0 \\
\left(\nabla_{X} J\right) Y=0
\end{array}\right.\right.
$$

i.e. the structure $\left(g, J,\left(\varphi_{i}, \xi_{i}, \eta_{i}\right)_{i=1}^{2}\right)$ is cosymplectic-Kählerian.

\section{Product $M^{4 m+2} \times \mathbb{R}^{2}$}

Let $\left(M^{4 m+2}, g, J,\left(\varphi_{i}, \xi_{i}, \eta_{i}\right)_{i=1}^{2}\right)$ be an almost Hermitian almost contact metric manifold. The product $\bar{M}=$ $M^{4 m+2} \times \mathbb{R}^{2}$, has dimension $4 n$ with $n=m+1$. We define for all $X, Y$ on $M$ and all $X^{\prime}, Y^{\prime}$ on $\mathbb{R}^{2}$ such that $X^{\prime}=f_{1} \frac{\partial}{\partial r_{1}}+f_{2} \frac{\partial}{\partial r_{2}}$ and $Y^{\prime}=h_{1} \frac{\partial}{\partial r_{1}}+h_{2} \frac{\partial}{\partial r_{2}}$ a three almost complex structures $\left(\tilde{J}_{i}\right)_{i=1}^{3}$ by;

$$
\begin{gathered}
\bar{J}_{1}\left(X, X^{\prime}\right)=\left(J X,-f_{2} \frac{\partial}{\partial r_{1}}+f_{1} \frac{\partial}{\partial r_{2}}\right), \\
\bar{J}_{2}\left(X, X^{\prime}\right)=\left(\varphi_{1} X-f_{1} \xi_{1}-f_{2} \xi_{2}, \eta_{1}(X) \frac{\partial}{\partial r_{1}}+\eta_{2}(X) \frac{\partial}{\partial r_{2}}\right),
\end{gathered}
$$




$$
\bar{J}_{3}\left(X, X^{\prime}\right)=\left(-\varphi_{2} X-f_{2} \xi_{1}+f_{1} \xi_{2}, \eta_{1}(X) \frac{\partial}{\partial r_{2}}-\eta_{2}(X) \frac{\partial}{\partial r_{1}}\right),
$$

and we define a Riemannian metric $\bar{g}$ by

$$
\bar{g}=g+d r_{1}^{2}+d r_{2}^{2}
$$

Proposition 4.1. The structure $\left(\bar{g},\left(\bar{J}_{i}\right)_{i=1}^{3}\right)$ constructed as above is an almost quaternionic metric .

Proof. Using (2.2) One can Obviously proof that.

By a direct computation using (2.1) and (2.3), one can check that the fundamental 2-form $\bar{\Omega}_{i}$ of $\left(\bar{J}_{i}, \bar{g}\right)$ for $i=\overline{1,2}$ is very simply as follows:

$$
\left\{\begin{array}{l}
\bar{\Omega}_{1}=\Omega-2\left(d r_{1} \wedge d r_{2}\right) \\
\bar{\Omega}_{2}=\phi_{1}-2\left(\eta_{1} \wedge d r_{1}+\eta_{2} \wedge d r_{2}\right), \\
\bar{\Omega}_{3}=-\phi_{2}-2\left(\eta_{1} \wedge d r_{2}-\eta_{2} \wedge d r_{1}\right),
\end{array}\right.
$$

Let $\nabla$ and $\bar{\nabla}$ denote the Riemannian connections of $g$ and $\bar{g}$ respectively. Now using (2.2) and ( 2.9 ) we get,

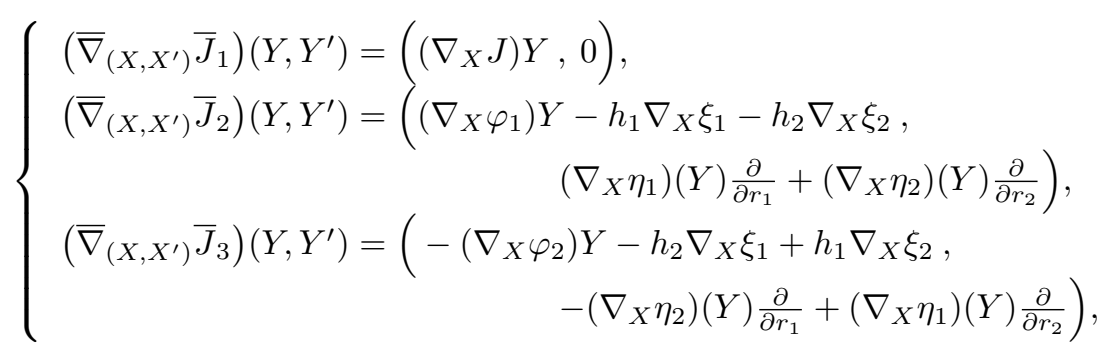

for all $X, Y$ on $M$ and all $X^{\prime}, Y^{\prime}$ on $\mathbb{R}^{2}$ such that $X^{\prime}=f_{1} \frac{\partial}{\partial r_{1}}+f_{2} \frac{\partial}{\partial r_{2}}$ and $Y^{\prime}=h_{1} \frac{\partial}{\partial r_{1}}+h_{2} \frac{\partial}{\partial r_{2}}$, and we can state the following:

Proposition 4.2. Let $\left(M^{4 m+2}, g, J,\left(\varphi_{i}, \xi_{i}, \eta_{i}\right)_{i=1}^{2}\right)$ be an almost Hermitian almost contact metric manifold. If $\left(\bar{g},\left(\bar{J}_{i}\right)_{i=1}^{3}\right)$ is an almost quaternionic metric structure on the manifold $\bar{M}=M \times \mathbb{R}^{2}$, constructed as above, then:

(a) $\bar{M}$ is almost hyperkählerian if, and only if, $M$ is almost cosymplectic-almost kählerian;

(b) $\bar{M}$ is hyperkählerian if, and only if, $M$ is cosymplectic-Kählerian.

Proof. Using the definition of almost kählerian structure and (4.1), The first assertion (a) is obviously. For (b), Using the definition of kählerian structure and hyperkählerian structure, one can adapt Proposition 3.2.

\section{Product $M^{4 m+2} \times M^{14 m^{\prime}+2}$}

Let $\left(M^{4 m+2}, g, J,\left(\varphi_{i}, \xi_{i}, \eta_{i}\right)_{i=1}^{2}\right),\left(M^{\prime 4 m^{\prime}+2}, g, J^{\prime},\left(\varphi_{i}^{\prime}, \xi_{i}^{\prime}, \eta_{i}^{\prime}\right)_{i=1}^{2}\right)$ two almost Hermitian almost contact metric manifolds. The product $\tilde{M}=M^{4 m+2} \times M^{\prime 4 m^{\prime}+2}$, has dimension $4 n\left(n=m+m^{\prime}+1\right)$. One defines a three almost complex structures $\left(\tilde{J}_{i}\right)_{i=1}^{3}$ by putting;

$$
\begin{gathered}
\tilde{J}_{1}=\left(J, J^{\prime}\right), \\
\tilde{J}_{2}=\left(\varphi_{2}+\eta_{1}^{\prime} \otimes \xi_{1}-\eta_{2}^{\prime} \otimes \xi_{2}, \varphi_{2}^{\prime}-\eta_{1} \otimes \xi_{1}^{\prime}+\eta_{2} \otimes \xi_{2}^{\prime}\right), \\
\tilde{J}_{3}=\left(\varphi_{1}-\eta_{1}^{\prime} \otimes \xi_{2}-\eta_{2}^{\prime} \otimes \xi_{1}, \varphi_{1}^{\prime}+\eta_{1} \otimes \xi_{2}^{\prime}+\eta_{2} \otimes \xi_{1}^{\prime}\right),
\end{gathered}
$$

and we define a Riemannian metric $\tilde{g}$ by

$$
\tilde{g}=g+g^{\prime}
$$

Proposition 5.1. The structure $\left(\tilde{g},\left(\tilde{J}_{i}\right)_{i=1}^{3}\right)$ constructed as above is an almost quaternionic metric structure. 
Proof. Obvious ( using (2.2)).

Let $\nabla, \nabla^{\prime}$ and $\tilde{\nabla}$ denote the Riemannian connections of $g, g^{\prime}$ and $\tilde{g}$ respectively. Now taking $X$ and $Y$ as vector fields tangent to $M$ and independent of $M^{\prime}$ and similarly for $X^{\prime}$ and $Y^{\prime}$ we give $\tilde{\nabla} \tilde{J}_{i}$ explicitly:

$$
\left\{\begin{aligned}
&\left(\tilde{\nabla}_{\left(X, X^{\prime}\right)} \tilde{J}_{1}\right)\left(Y, Y^{\prime}\right)=\left.\left(\nabla_{X} J\right) Y,\left(\nabla_{X^{\prime}}^{\prime} J^{\prime}\right) Y^{\prime}\right), \\
&\left(\tilde{\nabla}_{\left(X, X^{\prime}\right)} \tilde{J}_{2}\right)\left(Y, Y^{\prime}\right)=(\left(\nabla_{X} \varphi_{2}\right) Y+\eta_{1}^{\prime}\left(Y^{\prime}\right) \nabla_{X} \xi_{1}-\eta_{2}^{\prime}\left(Y^{\prime}\right) \nabla_{X} \xi_{2} \\
&+\left(\nabla_{X^{\prime}}^{\prime} \eta_{1}^{\prime}\right)\left(Y^{\prime}\right) \xi_{1}-\left(\nabla_{X}^{\prime}, \eta_{2}^{\prime}\right)\left(Y^{\prime}\right) \xi_{2}, \\
&\left(\nabla_{X^{\prime}}^{\prime} \varphi_{2}^{\prime}\right) Y^{\prime}-\eta_{1}(Y) \nabla_{X^{\prime}}^{\prime} \xi_{1}^{\prime}+\eta_{2}(Y) \nabla_{X^{\prime}}^{\prime} \xi_{2}^{\prime} \\
&-\left.\left(\nabla_{X} \eta_{1}\right)(Y) \xi_{1}^{\prime}+\left(\nabla_{X} \eta_{2}\right)(Y) \xi_{2}^{\prime}\right),(2) \\
&\left(\tilde{\nabla}_{\left(X, X^{\prime}\right)} \tilde{J}_{3}\right)\left(Y, Y^{\prime}\right)=(\left(\nabla_{X} \varphi_{1}\right) Y-\eta_{2}^{\prime}\left(Y^{\prime}\right) \nabla_{X} \xi_{1}-\eta_{1}^{\prime}\left(Y^{\prime}\right) \nabla_{X} \xi_{2} \\
&-\left(\nabla_{X^{\prime}}^{\prime} \eta_{2}^{\prime}\right)\left(Y^{\prime}\right) \xi_{1}-\left(\nabla_{X}^{\prime}, \eta_{1}^{\prime}\right)\left(Y^{\prime}\right) \xi_{2}, \\
&\left(\nabla_{X^{\prime}}^{\prime} \varphi_{1}^{\prime}\right) Y^{\prime}+\eta_{2}(Y) \nabla_{X}^{\prime}{ }^{\prime} \xi_{1}^{\prime}+\eta_{1}(Y) \nabla_{X^{\prime}}^{\prime} \xi_{2}^{\prime} \\
&\left.+\left(\nabla_{X} \eta_{2}\right)(Y) \xi_{1}^{\prime}+\left(\nabla_{X} \eta_{1}\right)(Y) \xi_{2}^{\prime}\right) .(3)
\end{aligned}\right.
$$

Suppose that $\tilde{\nabla} \tilde{J}_{i}=0$, by (1) we have $\nabla J=0$ and $\nabla^{\prime} J^{\prime}=0$. By (2) with $X^{\prime}=Y^{\prime}=0$ we get $\nabla \varphi_{2}=0$ and with $X=Y=0$ we get $\nabla^{\prime} \varphi_{2}^{\prime}=0$, with the same reasoning using (3) we find $\nabla \varphi_{1}=0$ and $\nabla^{\prime} \varphi_{1}^{\prime}=0$ i.e.

$$
\tilde{\nabla} \tilde{J}_{i}=0 \Rightarrow\left\{\begin{array}{l}
\nabla J=\nabla \varphi_{i}=0 \\
\nabla^{\prime} J^{\prime}=\nabla^{\prime} \varphi_{i}^{\prime}=0
\end{array}\right.
$$

conversely, knowing that $\nabla \varphi_{i}=0 \Rightarrow \nabla \eta_{i}=0 \Rightarrow \nabla \xi_{i}=0$ for $i=\overline{1,2}$ therefore if

$$
\left\{\begin{array}{l}
\nabla J=\nabla \varphi_{i}=0 \\
\nabla^{\prime} J^{\prime}=\nabla^{\prime} \varphi_{i}^{\prime}=0
\end{array}\right.
$$

then we get $\tilde{\nabla} \tilde{J}_{i}=0$ and we can state the following:

Theorem 5.1. The manifold $\tilde{M}$ is hyperkählerian if, and only if, $M$ and $M^{\prime}$ are two cosymplectic-Kählerian manifolds.

\section{Acknowledgements}

The author would like to thank the referees for their helpful suggestions, and Prof. T.Tshikuna-Matamba for his help during the preparation of this paper.

\section{References}

[1] Blair, D. E., Riemannian Geometry of Contact and Symplectic Manifolds. Progress in Mathematics Vol. 203, Birhauser, Boston,2002. bibitemBGM Boyer, C. P., Galicki, K. and Mann, B. M., Quaternionic reduction and Einstein manifolds. Comm. Anal. Geom. 1(2), (1993) pp.229-279.

[2] Calabi, E., Métriques kählériennes et fibrés holomorphes. Ann. Scient. Ec. Norm.Sup. 12(1979), pp. 269-294.

[3] Caprusi, M., Some remarks on the product of two almost contact manifolds. Al. I. Cuza, XXX, (1984) pp. 75-79 .

[4] Ishihara, S. and Konishi, M., Complex almost contact manifolds. Kodai Math. J. 3, (1980) pp.385-396

[5] Kuo, Y., On almost contact 3-structures. Tohoku Math. J. (2) 22 (1970), 325-332.

[6] Oubiña, J. A., A classification for almost contact structures. manuscript (1985).

[7] Satoh, H., Almost Hermitian structures on the tangent bundle. Procceedings of The Eleventh International Workshop on Differential.Geometry, Kyungpook Nat. Univ., Taegu, 11, (2007) pp.105-118 .

[8] Sharauddin, A. and Husain, S. I., Almost contact structures induced by a confotmal transformation. Pub. Inst. Math. 32(46), (1982), pp. 155-159.

[9] Tahara, M. and Watanabe, Y., Natural almost Hermitian, Hermitian and Kähler metrics on the tangent bundles. Math. J. Toyama Univ. 20(1997), pp. 149-160.

[10] Tshikuna-Matamba, T., Quelques classes des variétés métriques à 3- structures presque de contact. Ann. Univ. Craiova, Math. Comp. Sci. Ser. 31(1) (2004), pp. 94-101.

[11] Tshikuna-Matamba, T., Induced structures on the product of Riemannian manifolds. Int. Electron. J. Geo. Vol 4, (2011),pp. 15-25.

[12] Watson, B., Riemannian submersions and non-linear gauge field equations of general relativity, in Global Analysis-Analysis in Manifolds, (T.M. Rassias ed.) Teubner-Texte Math, Vol. 57, Teubner, Leipzig, (1983), 324-349. 
[13] Watanabe, Y., Almost Hermitian and Kähler structures on product manifolds. Proc. of the Thirteenth International Workshop on Diff. Geom. 13, (2009) 1-16.

[14] Watanabe, Y. and Hiroshi M., From Sasakian 3-structures to quaternionic geometry. Archivum Mathematicum, Vol. 34 (1998), No. 3, 379-386.

[15] Yano, K. and Kon, M., Structures on Manifolds. Series in Pure Math., Vol 3, World Sci.,1984.

\section{Affiliations}

\section{GHERICI BELDJILALI}

AdDress: Laboratoire de Physique Quantique de la matière et de Modélisation Mathématique (LPQ3M), Université de Mascara, Bp 305 route de Mamounia 29000, Mascara, Algeria

E-mail: Beldjilali29@gmail.com

\section{MOHAMED BELKHELFA}

AdDress: Present address: TAIBAH University, College of Science and Arts at al Ula, Department of Mathematics.

Laboratoire de Physique Quantique de la matière et de Modélisation Mathématique (LPQ3M), Université de Mascara, Bp 305 route de Mamounia 29000, Mascara, Algeria

E-mail: Belkhelfa@univ-mascara.dz or mohamed.belkhelfa@gmail.com 